\title{
The multiple Dirichlet product and the multiple Dirichlet series
}

\author{
TOMOKAZU ONOZUKA
}

III

\begin{abstract}
First, we define the multiple Dirichlet product and study the properties of it. From those properties, we obtain a zero-free region of a multiple Dirichlet series and a multiple Dirichlet series expression of the reciprocal of a multiple Dirichlet series.
\end{abstract}

\section{Introduction}

The Euler-Zagier multiple zeta function $\zeta_{E Z, k}\left(s_{1}, \ldots, s_{k}\right)$, the multiple zeta star function $\zeta_{k}^{*}\left(s_{1}, \ldots, s_{k}\right)$, and the Mordell-Tornheim multiple zeta function $\zeta_{M T, k}\left(s_{1}, \ldots, s_{k} ; s_{k+1}\right)$ are defined by

$$
\begin{aligned}
& \zeta_{E Z, k}\left(s_{1}, \ldots, s_{k}\right):=\sum_{0<m_{1}<m_{2}<\cdots<m_{k}} \frac{1}{m_{1}^{s_{1}} m_{2}^{s_{2}} \cdots m_{k}^{s_{k}}}, \\
& \zeta_{k}^{*}\left(s_{1}, \ldots, s_{k}\right):=\sum_{0<m_{1} \leq m_{2} \leq \cdots \leq m_{k}} \frac{1}{m_{1}^{s_{1}} m_{2}^{s_{2}} \cdots m_{k}^{s_{k}}}, \\
& \zeta_{M T, k}\left(s_{1}, \ldots, s_{k} ; s_{k+1}\right):=\sum_{m_{1}, \ldots, m_{k}=1}^{\infty} \frac{1}{m_{1}^{s_{1}} \cdots m_{k}^{s_{k}}\left(m_{1}+\cdots+m_{k}\right)^{s_{k+1}}},
\end{aligned}
$$

respectively, where $s_{i}(i=1, \ldots, k+1)$ are complex variables. Matsumoto [6] proved that the series (1.1) and (1.2) are absolutely convergent in

$$
\left\{\left(s_{1}, \ldots, s_{k}\right) \in \mathbb{C}^{k} \mid \Re\left(s_{k}(k-l+1)\right)>l \quad(l=1, \ldots, k)\right\}
$$

where $s_{k}(n)=s_{n}+s_{n+1}+\cdots+s_{k} \quad(n=1, \ldots, k)$. The series (1.3) is absolutely convergent in

$$
\left\{\left(s_{1}, \ldots, s_{k} ; s_{k+1}\right) \in \mathbb{C}^{k+1} \mid \Re\left(s_{l}\right)>1(l=1, \ldots, k), \Re\left(s_{k+1}\right)>0\right\} .
$$

2010 Mathematics Subject Classification: Primary 11M32; Secondary 11A25.

Key words and phrases: Multiple zeta function, Multiple zeta star function, Multiple Dirichlet series, Multiple Dirichlet product, Multiple Dirichlet convolution, Zero-free region.

The author was supported by JSPS KAKENHI Grant Number 13J00312. 
Akiyama, Egami and Tanigawa [1] and Zhao [11, independently of each other, proved the meromorphic continuation of the series (1.1) to the whole space. Akiyama, Egami and Tanigawa used the Euler-Maclaurin summation formula, while Zhao used generalized functions to prove the analytic continuation. Matsumoto [7] proved the meromorphic continuation of the series (1.3) to $\mathbb{C}^{k+1}$. The series (1.2) can be expressed by the sum of EulerZagier multiple zeta functions and the Riemann zeta function. (Note that the Riemann zeta function is one of Euler-Zagier multiple zeta functions.) For example, $\zeta_{2}^{*}$ and $\zeta_{3}^{*}$ can be expressed as a sum of Euler-Zagier multiple zeta functions as follows:

$$
\begin{aligned}
\zeta_{2}^{*}\left(s_{1}, s_{2}\right) & =\zeta_{E Z, 2}\left(s_{1}, s_{2}\right)+\zeta\left(s_{1}+s_{2}\right), \\
\zeta_{3}^{*}\left(s_{1}, s_{2}, s_{3}\right) & =\zeta_{E Z, 3}\left(s_{1}, s_{2}, s_{3}\right)+\zeta_{E Z, 2}\left(s_{1}+s_{2}, s_{3}\right)+\zeta_{E Z, 2}\left(s_{1}, s_{2}+s_{3}\right) \\
& +\zeta\left(s_{1}+s_{2}+s_{3}\right) .
\end{aligned}
$$

We can obtain the above expression by separating the sum of the series (1.2). From the above expression and the meromorphic continuation of EulerZagier multiple zeta functions, it follows that the multiple zeta star functions can be continued meromorphically to the whole space.

In this paper, as a generalization of the classical notion of Dirichlet series, we define multiple Dirichlet series by

$$
F\left(s_{1}, \ldots, s_{k} ; f\right):=\sum_{m_{1}, \ldots, m_{k}=1}^{\infty} \frac{f\left(m_{1}, \ldots, m_{k}\right)}{m_{1}^{s_{1}} \cdots m_{k}^{s_{k}}},
$$

where $f: \mathbb{N}^{k} \rightarrow \mathbb{C}$ and $\left(s_{1}, \ldots, s_{k}\right)$ is in the region of absolute convergence for $F\left(s_{1}, \ldots, s_{k} ; f\right)$. The series (1.5) is a generalization of the series (1.1), (1.2) and (1.3). De la Bretéche [4] treated function (1.5) in the case $f\left(m_{1}, \ldots, m_{k}\right)>0$.

In this paper, we study the multiple Dirichlet series defined by (1.5). In Section 2, we consider a ring of multiple arithmetic functions with the usual addition + and the multiple Dirichlet product $*$. In the case of one variable, Cashwell and Everett [3] proved that the ring is a UFD. In a way similar to that in [3], we find that the ring is also a UFD in the multivariable case. In Section 3, we treat the multiple Dirichlet series (1.5) and prove the main theorem (Theorem 3.5). The main theorem has two statements. In the first statement, we state about a zero-free region of $F\left(s_{1}, \ldots, s_{k} ; f\right)$. In the second statement, we state that the reciprocal of $F\left(s_{1}, \ldots, s_{k} ; f\right)$ has a multiple Dirichlet series expression $F\left(s_{1}, \ldots, s_{k} ; f^{-1}\right)$. Finally, by using the 
main theorem, we get a multiple Dirichlet series expression of the reciprocal of $\zeta_{k}^{*}\left(s_{1}, \ldots, s_{k}\right)$.

The author would like to express his thanks to Professor Kohji Matsumoto, Professor László Tóth, and Miss Ade Irma Suriajaya for their valuable advice and comments.

\section{The ring of multiple arithmetic functions}

We call $f: \mathbb{N}^{k} \longrightarrow \mathbb{C}$ a multiple (k-tuple) arithmetic function, and we define the set of $k$-tuple arithmetic functions by

$$
\Omega=\Omega_{k}:=\left\{f \mid f: \mathbb{N}^{k} \longrightarrow \mathbb{C}\right\}
$$

We define the set $U$ by;

$$
U=U_{k}:=\{f \in \Omega \mid f(1, \ldots, 1) \neq 0\}
$$

We use bold letters to express $k$-tuple of integers like $\boldsymbol{a}=\left(a_{1}, \ldots, a_{k}\right)$. In particular, 1 means $(1, \ldots, 1)$. Moreover we define the product of two $k$-tuple of integers $\boldsymbol{a} \cdot \boldsymbol{b}$ by $\left(a_{1} b_{1}, \ldots, a_{k} b_{k}\right)$.

Definition 2.1. For $f, g \in \Omega$ and $\boldsymbol{n} \in \mathbb{N}^{k}$, we define the multiple Dirichlet product $*$ by

$$
(f * g)(\boldsymbol{n})=\sum_{\substack{\boldsymbol{a} \cdot \boldsymbol{b}=\boldsymbol{n} \\ \boldsymbol{a}, \boldsymbol{b} \in \mathbb{N}^{k}}} f(\boldsymbol{a}) g(\boldsymbol{b})
$$

If $k=1$, the multiple Dirichlet product is the well-known Dirichlet product. Hence the above product is a generalization of the Dirichlet product. In the present section, our purpose is to study the properties of the multiple Dirichlet product. The properties were studied by some mathematicians. In [10], Tóth described the details of such studies. In particular, if we define an addition as $(f+g)(\boldsymbol{n}):=f(\boldsymbol{n})+g(\boldsymbol{n})$, he mentioned that $\left(\Omega_{k},+, *\right)$ is an integral domain with the identity function $I$ which is defined by

$$
I(\boldsymbol{n}):= \begin{cases}1 & (\boldsymbol{n}=\mathbf{1}) \\ 0 & \text { (otherwise) }\end{cases}
$$

and its unit group is $U$. For $f \in U, f^{-1}$ denotes the inverse function of $f$ with respect to the multiple Dirichlet product $*$. Then $f^{-1}$ is given by the following lemma. 
Lemma 2.2. For each $f \in U, f^{-1} \in U$ can be constructed recursively as follows;

$$
\begin{aligned}
f^{-1}(\mathbf{1}) & =\frac{1}{f(\mathbf{1})}, \\
f^{-1}(\boldsymbol{n}) & =-\frac{1}{f(\mathbf{1})} \sum_{\substack{\boldsymbol{a} \cdot \boldsymbol{b}=\boldsymbol{n} \\
\boldsymbol{b} \neq \boldsymbol{n}}} f(\boldsymbol{a}) f^{-1}(\boldsymbol{b}) \quad(\boldsymbol{n} \neq 1) .
\end{aligned}
$$

Proof. In the case $n_{1}+\cdots+n_{k}=k$, i.e. $\boldsymbol{n}=\mathbf{1}$, we have

$$
\left(f * f^{-1}\right)(\mathbf{1})=f(\mathbf{1}) f^{-1}(\mathbf{1})=1
$$

Next, let $d>k$, and assume that $f^{-1}(\boldsymbol{n})$ are determined for all $\boldsymbol{n} \in \mathbb{N}^{k}$ which satisfies $n_{1}+\cdots+n_{k}<d$. Then for $\boldsymbol{n} \in \mathbb{N}^{k}$ which satisfies $n_{1}+\cdots+n_{k}=d$, we have

$$
\begin{aligned}
\left(f * f^{-1}\right)(\boldsymbol{n}) & =\sum_{\boldsymbol{a} \cdot \boldsymbol{b}=\boldsymbol{n}} f(\boldsymbol{a}) f^{-1}(\boldsymbol{b}) \\
& =f(\mathbf{1}) f^{-1}(\boldsymbol{n})+\sum_{\substack{\boldsymbol{a} \cdot \boldsymbol{b}=\boldsymbol{n} \\
\boldsymbol{b} \neq \boldsymbol{n}}} f(\boldsymbol{a}) f^{-1}(\boldsymbol{b})=0 .
\end{aligned}
$$

In this section, let us consider $\left(\Omega_{k},+, *\right)$ and prove that $\left(\Omega_{k},+, *\right)$ is a UFD.

First, we define a norm $N: \Omega \longrightarrow \mathbb{Z}_{\geq 0}$ by

$$
N(f)= \begin{cases}0 & (f=0), \\ \min _{\boldsymbol{n} \in \mathbb{N}^{k}}\left\{n_{1} \cdots n_{k} \mid f(\boldsymbol{n}) \neq 0\right\} & (f \neq 0) .\end{cases}
$$

By the definition of the norm, we can show that $N(f)=1$ if and only if $f \in U$ holds.

Theorem 2.3. For $f, g \in \Omega$, we have

$$
N(f * g)=N(f) N(g)
$$

Proof. In the case $f=0$ or $g=0$, since $f * g=0$, we have $N(f *$ $g)=N(f) N(g)=0$. Hence we consider the case $f, g \neq 0$. If $n_{1} \cdots n_{k}<$ $N(f) N(g)$, then we have

$$
(f * g)(\boldsymbol{n})=\sum_{\boldsymbol{a} \cdot \boldsymbol{b}=\boldsymbol{n}} f(\boldsymbol{a}) g(\boldsymbol{b})=0
$$


since $a_{1} \cdots a_{k}<N(f)$ or $b_{1} \cdots b_{k}<N(g)$. Therefore it is sufficient to find $\boldsymbol{n}$ such that $(f * g)(\boldsymbol{n}) \neq 0$ and $n_{1} \cdots n_{k}=N(f) N(g)$. We define $\left(M_{1}, \ldots, M_{k}\right),\left(L_{1}, \ldots, L_{k}\right) \in \mathbb{N}^{k}$ as follows;

$$
\begin{aligned}
M_{1} & :=\min \left\{m_{1} \mid f\left(m_{1}, \ldots, m_{k}\right) \neq 0, m_{1} \cdots m_{k}=N(f)\right\}, \\
M_{2} & :=\min \left\{m_{2} \mid f\left(M_{1}, m_{2}, \ldots, m_{k}\right) \neq 0, M_{1} m_{2} \cdots m_{k}=N(f)\right\}, \\
& \vdots \\
M_{k} & :=\min \left\{m_{k} \mid f\left(M_{1}, \ldots, M_{k-1}, m_{k}\right) \neq 0, M_{1} \cdots M_{k-1} m_{k}=N(f)\right\}, \\
L_{1} & :=\min \left\{l_{1} \mid g\left(l_{1}, \ldots, l_{k}\right) \neq 0, l_{1} \cdots l_{k}=N(g)\right\} \\
L_{2} & :=\min \left\{l_{2} \mid g\left(L_{1}, l_{2}, \ldots, l_{k}\right) \neq 0, L_{1} l_{2} \cdots l_{k}=N(g)\right\}, \\
& \vdots \\
L_{k} & :=\min \left\{l_{k} \mid g\left(L_{1}, \ldots, L_{k-1}, l_{k}\right) \neq 0, L_{1} \cdots L_{k-1} l_{k}=N(g)\right\} .
\end{aligned}
$$

Next we prove

$$
\begin{aligned}
(f * g)(\boldsymbol{M} \cdot \boldsymbol{L}) & =\sum_{\boldsymbol{a} \cdot \boldsymbol{b}=\boldsymbol{M} \cdot \boldsymbol{L}} f(\boldsymbol{a}) g(\boldsymbol{b}) \\
& =f(\boldsymbol{M}) g(\boldsymbol{L}) \neq 0 .
\end{aligned}
$$

For $\boldsymbol{a} \neq \boldsymbol{M}$ with $a_{1} \cdots a_{k}=N(f)$, there exists an index $i$ such that $a_{i} \neq M_{i}$ and $a_{j}=M_{j}$ for all $j<i$. If $a_{i}<M_{i}$, by the definition of $M_{i}, f(\boldsymbol{a})=0$. If $a_{i}>M_{i}$, then $b_{i}<L_{i}$ and $b_{j}=L_{j}$ for $j<i$, so $g(\boldsymbol{B})=0$. Hence (2.1) holds.

Hence we have $N(f * g)=N(f) N(g)$.

Next, we define an equivalence relation among functions belonging to $\Omega$. For $f, g \in \Omega$, we write $f \sim g$ and say $f$ is equivalent to $g$ if there exists a function $\varepsilon \in U$ such that $f=\varepsilon * g$. Also we write $f \mid g$ if $g=f * h$ for some $h \in \Omega$.

Theorem 2.4. Suppose $f, g \in \Omega$. Then $f$ is equivalent to $g$, if and only if $f \mid g$ and $g \mid f$.

Proof. Assume $f \sim g$. Then there exists a function $\varepsilon \in U$ such that $f=$ $\varepsilon * g$, that is, $g \mid f$. Since $\varepsilon \in U, \varepsilon^{-1} * f=g$ holds. This means $f \mid g$.

Next, we assume $f \mid g$ and $g \mid f$. If $f=0$, then $g$ is also 0 . Therefore $f=\varepsilon * g$ holds for any $\varepsilon \in U$. Hence we are only left to consider the case $f \neq 0$. We see that $f \mid g$ and $g \mid f$ if and only if there exist $\alpha, \beta \in \Omega$ such that $f=\alpha * g$ and $g=\beta * f$, and if so, then $N(f)=N(\alpha) N(g)$ and $N(g)=N(\beta) N(f)$. From the above equations, it follows that $N(\alpha) N(\beta)=1$. It implies $\alpha, \beta \in U$. Hence $f$ is equivalent to $g$. 
For $p \in \Omega_{k} \backslash U$, we call $p$ prime if $p=f * g$ implies $f \in U$ or $g \in U$. We define $P_{k}=P$ to be the set of all prime functions. If $N(f)$ is prime in $\mathbb{N}$, then $f$ is prime in $\Omega$. Hence we can find infinitely many primes. A multiple arithmetic function $f \in \Omega$ is said to be composite if $f$ satisfies $f \neq 0, f \notin U$, and $f \notin P$. If $f \sim g$, then $f \in P$ implies $g \in P$. This property indicates that the equivalence relation $\sim$ preserves primitivity. The same property holds for $0, U$, and composite functions, respectively.

Next, we show that each non-zero function $f \in \Omega \backslash U$ can be decomposed into a finite product of prime functions. In the case $f \in P$, the function $f$ itself is the finite product of prime functions. Hence we consider the case $f \notin$ $P$. By the assumption, there exist multiple arithmetic functions $g, h \in \Omega \backslash U$ such that $f=g * h$. Then the inequalities $1<N(g), N(h)<N(f)$ hold. If both of $g$ and $h$ are primes, then $g * h$ is the prime factorization of $f$. If $g$ is a composite function, then there exist multiple arithmetic functions $g_{1}, g_{2} \in$ $\Omega \backslash U$ such that $g=g_{1} * g_{2}$, and the inequalities $1<N\left(g_{1}\right), N\left(g_{2}\right)<N(g)$ hold. Repeating the above algorithm, we can obtain the prime factorization of $f$. Because the norm is a non-negative integer, the product is finite.

Let $P_{\mathbb{N}}$ be the set of all prime numbers in $\mathbb{N}$. We define a set $\mathcal{P}_{k}=\mathcal{P}$ by

$$
\mathcal{P}_{k}=\mathcal{P}:=\left\{(1, \ldots, \underset{j}{1, p}, 1, \ldots, 1) \in \mathbb{N}^{k} \mid p \in P_{\mathbb{N}}, 1 \leq j \leq k\right\}
$$

We take a bijection $S: \mathbb{N} \longrightarrow \mathcal{P}$. Since $\mathcal{P}$ is a countably infinite set, we can take the bijection $S$. We put $\boldsymbol{p}_{j}:=S(j)$. Then each $\boldsymbol{m} \in \mathbb{N}^{k}$ can be decomposed into a finite product of $\mathcal{P}$. We define maps $\alpha_{j}(j \in \mathbb{N})$ as follows;

$$
\boldsymbol{m}=: \boldsymbol{p}_{1}^{\alpha_{1}(\boldsymbol{m})} \cdot \boldsymbol{p}_{2}^{\alpha_{2}(\boldsymbol{m})} \ldots
$$

The definition of $\alpha_{j}$ implies the equation $\alpha_{j}(\boldsymbol{a} \cdot \boldsymbol{b})=\alpha_{j}(\boldsymbol{a})+\alpha_{j}(\boldsymbol{b})$. By using $\alpha_{j}$, we define a map $R: \Omega \longrightarrow \mathbb{C}_{\omega}:=\mathbb{C}\left\{x_{1}, x_{2}, \ldots\right\}$ by $R(f)=$ $\sum_{m_{1}, \ldots, m_{k}=1}^{\infty} f(\boldsymbol{m}) x_{1}^{\alpha_{1}(\boldsymbol{m})} x_{2}^{\alpha_{2}(\boldsymbol{m})} \ldots$ for $f \in \Omega$, where $\mathbb{C}_{\omega}$ is the set of the infinite series of the above form, where each term has only a finite number of $x_{j}$. Note that although the series $R(f)$ contains $k$, the set $\mathbb{C}_{\omega}$ does not depend on $k$.

Lemma 2.5. The map $R$ is an isomorphism.

Proof. The equation $R(f+g)=R(f)+R(g)$ is trivial. In addition, we 
have

$$
\begin{aligned}
R(f * g) & =\sum_{m_{1}, \ldots, m_{k}=1}^{\infty} \sum_{\boldsymbol{a} \cdot \boldsymbol{b}=\boldsymbol{m}} f(\boldsymbol{a}) g(\boldsymbol{b}) x_{1}^{\alpha_{1}(\boldsymbol{a})+\alpha_{1}(\boldsymbol{b})} x_{2}^{\alpha_{2}(\boldsymbol{a})+\alpha_{2}(\boldsymbol{b})} \ldots \\
& =\left(\sum_{\boldsymbol{a}} f(\boldsymbol{a}) x_{1}^{\alpha_{1}(\boldsymbol{a})} x_{2}^{\alpha_{2}(\boldsymbol{a})} \ldots\right)\left(\sum_{\boldsymbol{b}} g(\boldsymbol{b}) x_{1}^{\alpha_{1}(\boldsymbol{b})} x_{2}^{\alpha_{2}(\boldsymbol{b})} \ldots\right) \\
& =R(f) R(g) .
\end{aligned}
$$

Hence $R$ is homomorphism.

Next, we show that $R$ is a bijection. Trivially $f \neq g$ implies $R(f) \neq R(g)$. For any $A \in \mathbb{C}_{\omega}$, we can constitute a multiple arithmetic function $f_{A} \in \Omega$ by using coefficients of $A$ such that $R\left(f_{A}\right)=A$.

In the case of one variable, Cashwell and Everett [3] proved that the ring $\left(\Omega_{1},+, *\right)$ is a UFD by first showing that $\Omega_{1}$ is isomorphic to $\mathbb{C}_{\omega}$ and then showing that $\left(\mathbb{C}_{\omega},+, \times\right)$ is a UFD. Here, for the multivariable case, we use Lemma 2.5 and the result of Cashwell and Everett that $\left(\mathbb{C}_{\omega},+, \times\right)$ is a UFD, and we obtain the following theorem.

Theorem 2.6. $\left(\Omega_{k},+, *\right)$ is a UFD.

In addition to the above theorem, Lemma 2.5 implies an another theorem. Lemma 2.5 states that $\Omega_{k}$ is isomorphic to $\mathbb{C}_{\omega}$ for all $k \in \mathbb{N}$. This fact means that $\Omega_{k}$ is isomorphic to $\Omega_{l}$ for $k, l \in \mathbb{N}$.

Theorem 2.7. All of $\Omega_{k}$ are isomorphic, i.e. $\Omega_{k} \cong \Omega_{l}$ holds for all $k, l \in \mathbb{N}$.

\section{$3 \quad$ The multiple Dirichlet series}

In this section, we consider the zero-free region of the multiple Dirichlet series and the reciprocal of the multiple Dirichlet series by using the notion of multiple Dirichlet product. The following theorem is a basic property of the multiple Dirichlet series.

Theorem 3.1. ([10], Proposition 10) For $f, g \in \Omega_{k}$, we have

$$
\begin{aligned}
& F\left(s_{1}, \ldots, s_{k} ; f\right)+F\left(s_{1}, \ldots, s_{k} ; g\right)=F\left(s_{1}, \ldots, s_{k} ; f+g\right), \\
& F\left(s_{1}, \ldots, s_{k} ; f\right) F\left(s_{1}, \ldots, s_{k} ; g\right)=F\left(s_{1}, \ldots, s_{k} ; f * g\right),
\end{aligned}
$$

where $\left(s_{1}, \ldots, s_{k}\right)$ lies in the region of absolute convergence for the series $F\left(s_{1}, \ldots, s_{k} ; f\right)$ and $F\left(s_{1}, \ldots, s_{k} ; g\right)$. 
The multiple Dirichlet product

Corollary 3.2. Let $f \in U$. If $F\left(s_{1}, \ldots, s_{k} ; f\right)$ and $F\left(s_{1}, \ldots, s_{k} ; f^{-1}\right)$ are absolutely convergent on $R \subset \mathbb{C}^{k}$, then $F\left(s_{1}, \ldots, s_{k} ; f\right)$ has no zeros on $R$.

Proof. Let $\left(s_{1}, \ldots, s_{k}\right) \in R$. Then by applying Theorem 3.1 , we have

$$
F\left(s_{1}, \ldots, s_{k} ; f\right) F\left(s_{1}, \ldots, s_{k} ; f^{-1}\right)=F\left(s_{1}, \ldots, s_{k} ; I\right)=1 .
$$

To find the zero-free region of the series $F\left(s_{1}, \ldots, s_{k} ; f\right)$, we have to find the region of absolute convergence $R \subset \mathbb{C}^{k}$, and to find the region $R$, we have to evaluate $f^{-1}$. For this purpose, we prepare the following lemma.

Lemma 3.3. For $\alpha>1$, we have

$$
\sum_{d \mid n} d^{\alpha} \leq \zeta(\alpha) n^{\alpha}
$$

Proof. We have

$$
\sum_{d \mid n} d^{\alpha}=n^{\alpha} \sum_{d \mid n}\left(\frac{d}{n}\right)^{\alpha}=n^{\alpha} \sum_{d \mid n} \frac{1}{d^{\alpha}} \leq \zeta(\alpha) n^{\alpha} .
$$

By the above lemma, we can evaluate the function $f^{-1}$.

Theorem 3.4. Let $f \in U$ satisfy the condition that there exist constants $C>0$ and $r_{1}, \ldots, r_{k} \in \mathbb{R}$ such that $|f(\boldsymbol{n})| \leq C n_{1}^{r_{1}} n_{2}^{r_{2}} \cdots n_{k}^{r_{k}}$ for $\boldsymbol{n} \neq \mathbf{1}$. We put $\alpha_{j}>1+r_{j}(j=1, \ldots, k)$ satisfying $\zeta\left(\alpha_{1}-r_{1}\right) \zeta\left(\alpha_{2}-r_{2}\right) \cdots \zeta\left(\alpha_{k}-r_{k}\right) \leq$ $1+|f(\mathbf{1})| / C$. Then we have

$$
\left|f^{-1}(\boldsymbol{n})\right| \leq \frac{n_{1}^{\alpha_{1}} n_{2}^{\alpha_{2}} \cdots n_{k}^{\alpha_{k}}}{|f(\mathbf{1})|}
$$

Proof. We use induction on $n_{1}+\cdots+n_{k}$. In the case $n_{1}+\cdots+n_{k}=k$, i.e. $\boldsymbol{n}=\mathbf{1}$, then $f^{-1}(\mathbf{1})=1 / f(\mathbf{1})$, so we have

$$
\left|f^{-1}(\mathbf{1})\right|=\frac{1}{|f(\mathbf{1})|}
$$

Next, let $d>k$, and assume that $\left|f^{-1}(\boldsymbol{n})\right| \leq n_{1}^{\alpha_{1}} n_{2}^{\alpha_{2}} \cdots n_{k}^{\alpha_{k}} /|f(\mathbf{1})|$ for all $\boldsymbol{n} \in \mathbb{N}^{k}$ which satisfies $n_{1}+\cdots+n_{k}<d$. Then for $\boldsymbol{n} \in \mathbb{N}^{k}$ which satisfies 


$$
\begin{aligned}
n_{1}+\cdots & +n_{k}=d, \text { by using Lemma 3.3, we have } \\
\left|f^{-1}(\boldsymbol{n})\right| & \leq\left|\frac{1}{f(\mathbf{1})}\right| \sum_{\substack{\boldsymbol{a} \cdot \boldsymbol{b}=\boldsymbol{n} \\
\boldsymbol{a} \boldsymbol{b} \in \mathbb{N}^{k} \\
\boldsymbol{b} \neq \boldsymbol{n}}}|f(\boldsymbol{a})|\left|f^{-1}(\boldsymbol{b})\right| \\
& \leq \frac{C}{|f(\mathbf{1})|^{2}} \sum_{\substack{\boldsymbol{a} \cdot \boldsymbol{b}=\boldsymbol{n} \\
\boldsymbol{b} \neq \boldsymbol{n}}} a_{1}^{r_{1}} b_{1}^{\alpha_{1}} \cdots a_{k}^{r_{k}} b_{k}^{\alpha_{k}} \\
& =\frac{C}{|f(\mathbf{1})|^{2}}\left\{n_{1}^{r_{1}} \cdots n_{k}^{r_{k}}\left(\sum_{b_{1} \mid n_{1}} b_{1}^{\alpha_{1}-r_{1}}\right) \cdots\left(\sum_{b_{k} \mid n_{k}} b_{k}^{\alpha_{k}-r_{k}}\right)-n_{1}^{\alpha_{1}} \cdots n_{k}^{\alpha_{k}}\right\} \\
& \leq \frac{C}{|f(\mathbf{1})|^{2}}\left(\zeta\left(\alpha_{1}-r_{1}\right) n_{1}^{\alpha_{1}} \ldots \zeta\left(\alpha_{k}-r_{k}\right) n_{k}^{\alpha_{k}}-n_{1}^{\alpha_{1}} n_{2}^{\alpha_{2}} \cdots n_{k}^{\alpha_{k}}\right) \\
& \leq \frac{n_{1}^{\alpha_{1}} n_{2}^{\alpha_{2}} \cdots n_{k}^{\alpha_{k}}}{|f(\mathbf{1})|} .
\end{aligned}
$$

Finally, we obtain the main theorem.

Theorem 3.5. Let $f$ and $\alpha_{1}, \ldots, \alpha_{k}$ satisfy the conditions in Theorem 3.4 . Then $F\left(s_{1}, \ldots, s_{k} ; f\right)$ and $F\left(s_{1}, \ldots, s_{k} ; f^{-1}\right)$ have no zeros in the region

$$
\left\{\left(s_{1}, \ldots, s_{k}\right) \in \mathbb{C}^{k} \mid \Re\left(s_{j}\right)>1+\alpha_{j}(j=1, \cdots, k)\right\}
$$

Moreover, in the same region $F\left(s_{1}, \ldots, s_{k} ; f\right)$ and $F\left(s_{1}, \ldots, s_{k} ; f^{-1}\right)$ satisfy the relation

$$
\left(F\left(s_{1}, \ldots, s_{k} ; f\right)\right)^{-1}=F\left(s_{1}, \ldots, s_{k} ; f^{-1}\right) .
$$

Proof. Since $f(\boldsymbol{n}) \ll n_{1}^{r_{1}} n_{2}^{r_{2}} \cdots n_{k}^{r_{k}}, F\left(s_{1}, \ldots, s_{k} ; f\right)$ is convergent absolutely in

$$
\left\{\left(s_{1}, \ldots, s_{k}\right) \in \mathbb{C}^{k} \mid \Re\left(s_{j}\right)>1+r_{j}(j=1, \ldots, k)\right\} .
$$

Since $f^{-1}(\boldsymbol{n}) \ll n_{1}^{\alpha_{1}} \cdots n_{k}^{\alpha_{k}}$ by Theorem 3.4, $F\left(s_{1}, \ldots, s_{k} ; f^{-1}\right)$ is convergent absolutely in

$$
\left\{\left(s_{1}, \ldots, s_{k}\right) \in \mathbb{C}^{k} \mid \Re\left(s_{j}\right)>1+\alpha_{j}(j=1, \ldots, k)\right\}
$$

Hence we obtain the theorem by using Theorem 3.2 . 
Next, we consider a restricted multiple Dirichlet series. To express the series (1.1), (1.2) and (1.3), we define three functions as follows;

$$
\begin{aligned}
& u_{E Z}(\boldsymbol{n}):= \begin{cases}1 & \left(n_{1}<n_{2}<\cdots<n_{k}\right), \\
0 & (\text { otherwise }),\end{cases} \\
& u^{*}(\boldsymbol{n}):= \begin{cases}1 & \left(n_{1} \leq n_{2} \leq \cdots \leq n_{k}\right), \\
0 & (\text { otherwise })\end{cases} \\
& u_{M T}(\boldsymbol{n}):= \begin{cases}1 & \left(n_{k+1}=n_{1}+n_{2}+\cdots+n_{k}\right), \\
0 & \text { (otherwise) } .\end{cases}
\end{aligned}
$$

Then the series (1.1), (1.2) and (1.3) can be expressed by

$$
\begin{aligned}
& \zeta_{E Z, k}\left(s_{1}, \ldots, s_{k}\right)=F\left(s_{1}, \ldots, s_{k} ; u_{E Z}\right) \\
& \zeta_{k}^{*}\left(s_{1}, \ldots, s_{k}\right)=F\left(s_{1}, \ldots, s_{k} ; u^{*}\right) \\
& \zeta_{M T, k}\left(s_{1}, \ldots, s_{k} ; s_{k+1}\right)=F\left(s_{1}, \ldots, s_{k+1} ; u_{M T}\right) .
\end{aligned}
$$

In [2], Akiyama and Ishikawa treated the multiple $L$-function which is defined by

$$
L_{k}\left(s_{1}, \ldots, s_{k} \mid \chi_{1}, \ldots, \chi_{k}\right):=\sum_{m_{1}<m_{2}<\cdots<m_{k}} \frac{\chi_{1}\left(m_{1}\right) \cdots \chi_{k}\left(m_{k}\right)}{m_{1}^{s_{1}} m_{2}^{s_{2}} \cdots m_{k}^{s_{k}}},
$$

where $\chi_{j}$ are Dirichlet characters. This is a generalization of the series (1.1). Moreover in [8], Matsumoto and Tanigawa treated the series

$$
\sum_{m_{1}, \ldots, m_{k}=1}^{\infty} \frac{a_{1}\left(m_{1}\right) a_{2}\left(m_{2}\right) \cdots a_{k}\left(m_{k}\right)}{m_{1}^{s_{1}}\left(m_{1}+m_{2}\right)^{s_{2}} \cdots\left(m_{1}+\cdots+m_{k}\right)^{s_{k}}}
$$

where series $\sum_{m=1}^{\infty} a_{j}(m) / m^{s}(1 \leq j \leq k)$ satisfy good conditions. This is also a generalization of the series (1.1).

To consider such series, we generalize the series (1.1) as follows;

$$
\sum_{0<m_{1}<m_{2}<\cdots<m_{k}} \frac{f\left(m_{1}, \ldots, m_{k}\right)}{m_{1}^{s_{1}} m_{2}^{s_{2}} \cdots m_{k}^{s_{k}}}
$$

This series is a restriction of the multiple Dirichlet series (1.5). To treat the restricted multiple Dirichlet series, we define three sets by

$\Omega_{E Z}:=\left\{f \in \Omega \mid f(\boldsymbol{n})=0\right.$ for $\boldsymbol{n}$ which does not satisfy $\left.n_{1}<\cdots<n_{k}\right\}$,

$\Omega^{*}:=\left\{f \in \Omega \mid f(\boldsymbol{n})=0\right.$ for $\boldsymbol{n}$ which does not satisfy $\left.n_{1} \leq \cdots \leq n_{k}\right\}$, $\Omega_{M T}:=\left\{f \in \Omega \mid f(\boldsymbol{n})=0\right.$ for $\boldsymbol{n}$ which satisfies $\left.n_{k}<n_{1}+n_{2}+\cdots+n_{k-1}\right\}$. 
Theorem 3.6. The ring $\left(\Omega^{*},+, *\right)$ is a subring of the ring $(\Omega,+, *)$ which contains the unit group $\Omega^{*} \cap U$. The rings $\left(\Omega_{E Z},+, *\right)$ and $\left(\Omega_{M T},+, *\right)$ are subrings of the ring $(\Omega,+, *)$ which do not contain the unit group.

Proof. Trivially all of the sets are closed with respect to the addition + . Hence it is sufficient to consider the Dirichlet product $*$.

First we consider the set $\Omega^{*}$. Let $f, g \in \Omega^{*}$. For $\boldsymbol{n}$ which does not satisfy $n_{1} \leq \cdots \leq n_{k}$, we have

$$
(f * g)(\boldsymbol{n})=\sum_{\boldsymbol{a} \cdot \boldsymbol{b}=\boldsymbol{n}} f(\boldsymbol{a}) g(\boldsymbol{b})
$$

Then, for each pair of $\boldsymbol{a}$ and $\boldsymbol{b}$ which satisfies $\boldsymbol{a} \cdot \boldsymbol{b}=\boldsymbol{n}$, at least one of them does not satisfy the inequalities $a_{1} \leq \cdots \leq a_{k}$ and $b_{1} \leq \cdots \leq b_{k}$. It implies that $(f * g)(\boldsymbol{n})=0$. Therefore $f * g \in \Omega^{*}$ holds. Next we assume $f \in \Omega^{*} \cap U$. Suppose that there exists an $\boldsymbol{n}$ which satisfies $f^{-1}(\boldsymbol{n}) \neq 0$ and does not satisfy $n_{1} \leq \cdots \leq n_{k}$. We choose such $\boldsymbol{n}$ which satisfies the condition that $n_{1}+\cdots+n_{k}$ is the smallest. Similarly to the above proof, an equality

$$
f^{-1}(\boldsymbol{n})=-\frac{1}{f(\mathbf{1})} \sum_{\substack{\boldsymbol{a} \cdot \boldsymbol{b}=\boldsymbol{n} \\ \boldsymbol{b} \neq \boldsymbol{n}}} f(\boldsymbol{a}) f^{-1}(\boldsymbol{b})
$$

implies that $f^{-1}(\boldsymbol{n})=0$. It contradicts the assumption $f^{-1}(\boldsymbol{n}) \neq 0$. Hence $f^{-1} \in \Omega^{*} \cap U$ holds.

The statement that $\left(\Omega_{E Z},+, *\right)$ forms a subring can be proven similarly as above.

Next we prove the statement that $\left(\Omega_{M T},+, *\right)$ forms a subring. Let $f, g \in$ $\Omega_{M T}$. For $\boldsymbol{n}$, we have

$$
(f * g)(\boldsymbol{n})=\sum_{\boldsymbol{a} \cdot \boldsymbol{b}=\boldsymbol{n}} f(\boldsymbol{a}) g(\boldsymbol{b})
$$

If $\boldsymbol{a}$ and $\boldsymbol{b}$ satisfy the inequalities $a_{k} \geq a_{1}+\cdots+a_{k-1}$ and $b_{k} \geq b_{1}+\cdots+b_{k-1}$, the inequality

$$
\begin{aligned}
\left(a_{1} b_{1}+\cdots+a_{k-1} b_{k-1}\right)^{2} & \leq\left(a_{1}^{2}+\cdots+a_{k-1}^{2}\right)\left(b_{1}^{2}+\cdots+b_{k-1}^{2}\right) \\
& \leq\left(a_{1}+\cdots+a_{k-1}\right)^{2}\left(b_{1}+\cdots+b_{k-1}\right)^{2} \\
& \leq a_{k}^{2} b_{k}^{2}
\end{aligned}
$$

implies the inequality $n_{k} \geq n_{1}+\cdots+n_{k-1}$. Hence for $\boldsymbol{n}$ which satisfies $n_{k}<n_{1}+n_{2}+\cdots+n_{k-1}$, at least one of the inequalities $a_{k}<a_{1}+\cdots+a_{k-1}$ and $b_{k}<b_{1}+\cdots+b_{k-1}$ holds. Therefore the equation $(f * g)(\boldsymbol{n})=0$ is valid for $\boldsymbol{n}$ which satisfies $n_{k}<n_{1}+n_{2}+\cdots+n_{k-1}$. It implies that $f * g \in \Omega_{M T}$. 
By the above theorem, the product of two restricted multiple Dirichlet series is also a restricted multiple Dirichlet series, that is

$$
\begin{gathered}
\left(\sum_{0<m_{1}<\cdots<m_{k}} \frac{f\left(m_{1}, \ldots, m_{k}\right)}{m_{1}^{s_{1}} \cdots m_{k}^{s_{k}}}\right)\left(\sum_{0<n_{1}<\cdots<n_{k}} \frac{g\left(n_{1}, \ldots, n_{k}\right)}{n_{1}^{s_{1}} \cdots n_{k}^{s_{k}}}\right) \\
=\sum_{0<n_{1}<\cdots<n_{k}} \frac{(f * g)\left(n_{1}, \ldots, n_{k}\right)}{n_{1}^{s_{1}} \cdots n_{k}^{s_{k}}}, \\
\left(\sum_{0<m_{1} \leq \cdots \leq m_{k}} \frac{f\left(m_{1}, \ldots, m_{k}\right)}{m_{1}^{s_{1}} \cdots m_{k}^{s_{k}}}\right)\left(\sum_{0<n_{1} \leq \cdots \leq n_{k}} \frac{g\left(n_{1}, \ldots, n_{k}\right)}{n_{1}^{s_{1}} \cdots n_{k}^{s_{k}}}\right) \\
=\sum_{0<n_{1} \leq \cdots \leq n_{k}} \frac{(f * g)\left(n_{1}, \ldots, n_{k}\right)}{n_{1}^{s_{1}} \cdots n_{k}^{s_{k}}}, \\
\left.\sum_{m_{k} \geq m_{1}+\cdots+m_{k-1}} \frac{f\left(m_{1}, \ldots, m_{k}\right)}{m_{1}^{s_{1}} \cdots m_{k}^{s_{k}}}\right)\left(\begin{array}{c}
\left.\sum_{n_{k} \geq n_{1}+\cdots+n_{k-1}} \frac{g\left(n_{1}, \ldots, n_{k}\right)}{n_{1}^{s_{1}} \cdots n_{k}^{s_{k}}}\right) \\
=\sum_{n_{k} \geq n_{1}+\cdots+n_{k-1}} \frac{(f * g)\left(n_{1}, \ldots, n_{k}\right)}{n_{1}^{s_{1}} \cdots n_{k}^{s_{k}}} .
\end{array}\right.
\end{gathered}
$$

In addition, the inverse of a restricted multiple Dirichlet series is also a restricted multiple Dirichlet series, that is

$$
\left(\sum_{0<m_{1} \leq \cdots \leq m_{k}} \frac{f\left(m_{1}, \ldots, m_{k}\right)}{m_{1}^{s_{1}} \cdots m_{k}^{s_{k}}}\right)^{-1}=\sum_{0<m_{1} \leq \cdots \leq m_{k}} \frac{f^{-1}\left(m_{1}, \ldots, m_{k}\right)}{m_{1}^{s_{1}} \cdots m_{k}^{s_{k}}}
$$

for $f \in \Omega^{*} \cap U$.

In [7], Matsumoto defined the Apostol-Vu multiple zeta function by

$$
\zeta_{A V, k-1}\left(s_{1}, \ldots, s_{k-1} ; s_{k}\right):=\sum_{0<m_{1}<\cdots<m_{k-1}} \frac{1}{m_{1}^{s_{1}} \cdots m_{k-1}^{s_{k-1}}\left(m_{1}+\cdots+m_{k-1}\right)^{s_{k}}} .
$$

To express this series, we define a function $u_{A V}$ as follows;

$$
u_{A V}(\boldsymbol{n}):= \begin{cases}1 & \left(0<n_{1}<\cdots<n_{k} \text { and } n_{k}=n_{1}+n_{2}+\cdots+n_{k-1}\right), \\ 0 & \text { (otherwise). }\end{cases}
$$

Then $u_{A V} \in \Omega_{E Z} \cap \Omega_{M T}$ holds. If we define $\Omega_{A V}=\Omega_{E Z} \cap \Omega_{M T}$, then it follows from Theorem 3.6 that $\Omega_{A V}$ is a subring of the ring $(\Omega,+, *)$ which does not contain the unit group.

As mentioned in the introduction, the series (1.2) is absolutely convergent in the region (1.4). This fact improves Theorem 3.5. 
The multiple Dirichlet product

Theorem 3.7. Let $f \in \Omega^{*} \cap U$ and $\alpha_{1}, \ldots, \alpha_{k}$ satisfy the conditions in Theorem 3.4. Then $F\left(s_{1}, \ldots, s_{k} ; f\right)$ and $F\left(s_{1}, \ldots, s_{k} ; f^{-1}\right)$ have no zeros in the region

$$
\left\{\left(s_{1}, \ldots, s_{k}\right) \in \mathbb{C}^{k} \mid \Re\left(s_{k}(k-l+1)\right)>l+\alpha_{k}(k-l+1) \quad(l=1, \ldots, k)\right\}
$$

where $\alpha_{k}(l)=\alpha_{l}+\alpha_{l+1}+\cdots+\alpha_{k} \quad(l=1, \ldots, k)$. Moreover, in the same region $F\left(s_{1}, \ldots, s_{k} ; f\right)$ and $F\left(s_{1}, \ldots, s_{k} ; f^{-1}\right)$ satisfy the relation

$$
\left(F\left(s_{1}, \ldots, s_{k} ; f\right)\right)^{-1}=F\left(s_{1}, \ldots, s_{k} ; f^{-1}\right)
$$

Proof. Since $f(\boldsymbol{n}) \ll n_{1}^{r_{1}} n_{2}^{r_{2}} \cdots n_{k}^{r_{k}}, F\left(s_{1}, \ldots, s_{k} ; f\right)$ is absolutely convergent in

$$
\left\{\left(s_{1}, \ldots, s_{k}\right) \in \mathbb{C}^{k} \mid \Re\left(s_{k}(k-l+1)\right)>l+r_{k}(k-l+1) \quad(l=1, \ldots, k)\right\},
$$

where $r_{k}(l)=r_{l}+r_{l+1}+\cdots+r_{k} \quad(l=1, \ldots, k)$. Since $f^{-1}(\boldsymbol{n}) \ll n_{1}^{\alpha_{1}} \cdots n_{k}^{\alpha_{k}}$ by Theorem 3.4, $F\left(s_{1}, \ldots, s_{k} ; f^{-1}\right)$ is convergent absolutely in

$$
\left\{\left(s_{1}, \ldots, s_{k}\right) \in \mathbb{C}^{k} \mid \Re\left(s_{k}(k-l+1)\right)>l+\alpha_{k}(k-l+1) \quad(l=1, \ldots, k)\right\} .
$$

Hence we obtain the theorem by using Theorem 3.2 .

Applying Theorem 3.5, Theorem 3.7 and the identity theorem, we have the following corollary.

Corollary 3.8. $\zeta_{E Z, k}\left(s_{1}, \ldots, s_{k}\right)+1$ and $\zeta_{k}^{*}\left(s_{1}, \ldots, s_{k}\right)$ have no zeros in the region

$S:=\left\{\left(s_{1}, \ldots, s_{k}\right) \in \mathbb{C}^{k} \mid \Re\left(s_{k}(k-l+1)\right)>l+\alpha_{k}(k-l+1) \quad(l=1, \ldots, k)\right\}$

where $\alpha_{i}>1(i=1, \ldots, k)$ satisfy $\zeta\left(\alpha_{1}\right) \zeta\left(\alpha_{2}\right) \cdots \zeta\left(\alpha_{k}\right) \leq 2$. In the same region $S$, the reciprocal of the two functions have multiple Dirichlet series expressions

$$
\begin{aligned}
\left(\zeta_{E Z, k}\left(s_{1}, \ldots, s_{k}\right)+1\right)^{-1} & =F\left(s_{1}, \ldots, s_{k} ;\left(u_{E Z}+I\right)^{-1}\right), \\
\left(\zeta_{k}^{*}\left(s_{1}, \ldots, s_{k}\right)\right)^{-1} & =F\left(s_{1}, \ldots, s_{k} ;\left(u^{*}\right)^{-1}\right),
\end{aligned}
$$

respectively. Furthermore $F\left(s_{1}, \ldots, s_{k} ;\left(u_{E Z}+I\right)^{-1}\right)$ and $F\left(s_{1}, \ldots, s_{k} ;\left(u^{*}\right)^{-1}\right)$ are continued meromorphically to the whole space.

In the last corollary we obtained the zero free region of $\zeta_{k}^{*}$. This is not the best possible region. Similar to [9, equation (2)], we find that the region $S^{\prime}:=\left\{\left(s_{1}, \ldots, s_{k}\right) \in \mathbb{C}^{k} \mid \zeta_{k}^{*}\left(\Re s_{1}, \ldots, \Re s_{k}\right)<2, \Re\left(s_{k}(k-l+1)\right)>l \quad(l=1, \ldots, k)\right\}$ 
is also the zero free region, and this region has a point which is not contained in $S$, that is, $S^{\prime} \backslash S \neq \emptyset$. For example, when $k=2,\left(s_{1}, s_{2}\right)=(2,2) \notin S$, since for $\left(s_{1}, s_{2}\right) \in S, s_{2}$ needs to satisfy the condition $\Re\left(s_{2}\right)>2$. On the other hand, $(2,2) \in S^{\prime}$, since by [5, corollary 2.3] we have

$$
\zeta_{2}^{*}(2,2)=\zeta_{E Z, 2}(2,2)+\zeta(4)=\frac{\pi^{4}}{120}+\frac{\pi^{4}}{90}=\frac{7 \pi^{4}}{360}<2 .
$$

Hence $(2,2) \in S^{\prime} \backslash S$, so $S$ is not the best possible region. However, $S$ has a simpler expression than $S^{\prime}$. This is an advantage of Corollary 3.8 .

\section{References}

[1] S. Akiyama, S. Egami and Y. Tanigawa, Analytic continuation of multiple zeta-functions and their values at non-positive integers, Acta Arith, 98 (2001), 107-116.

[2] S. Akiyama and H. Ishikawa, On analytic continuation of multiple Lfunctions, Analytic Number Theory, C. Jia and K. Matsumoto, eds. (2002), 1-16.

[3] E. D. Cashwell and C. J. Everett, The ring of number-theoretic functions, Pacific J. Math. 9 (1959), 975-985.

[4] R. de la Bretéche, Estimation de sommes multiples de fonctions arithétiques, Compositio Mathematica 128 (2001), 261-298.

[5] M. Hoffman, Multiple harmonic series, Pacific J. Math., 152 (1992), $275-290$.

[6] K. Matsumoto, On analytic continuation of various multiple zetafunctions, Number Theory for the Millenium (Urbana, 2000), Vol. II, M. A. Bennett et. al. (eds.), A. K. Peters, Natick, MA, 2002, pp. 417440.

[7] K. Matsumoto, On Mordell-Tornheim and other multiple zeta-functions, In: Proc. Session in Analytic Number Theory and Diophantine Equations, (eds. D. R. Heath-Brown and B. Z. Moroz), Bonner Math. Schriften, 360, Bonn, 2003, n.25, 17pp.

[8] K. Matsumoto and Y. Tanigawa, The analytic continuation and the order estimate of multiple Dirichlet series, J. Théorie des Nombres de Bordeaux, 15 (2003), 267-274. 
[9] R. Spira, Zero-free regions of $\zeta^{(k)}(s)$, J. London Math. Soc., 40 (1965), 677-682.

[10] L. Tóth, Multiplicative arithmetic functions of several variables: a survey, preprint, arXiv:1310.7053.

[11] J. Zhao, Analytic continuation of multiple zeta functions, Proc. Amer. Math. Soc. 128 (2000), 1275-1283.

Graduate School of Mathematics

Nagoya University

Chikusa-ku, Nagoya 464-8602, Japan

E-mail: m11022v@math.nagoya-u.ac.jp 